\title{
Electric-Field Control of Exchange Bias in Multiferroic Epitaxial Heterostructures
}

\author{
V. Laukhin, ${ }^{1,2}$ V. Skumryev, ${ }^{2,3}$ X. Martí, ${ }^{1}$ D. Hrabovsky, ${ }^{1}$ F. Sánchez, ${ }^{1}$ M. V. García-Cuenca, ${ }^{4}$ C. Ferrater, ${ }^{4}$ M. Varela, ${ }^{4}$ \\ U. Lüders, ${ }^{5}$ J. F. Bobo, ${ }^{5}$ and J. Fontcuberta ${ }^{1}$ \\ ${ }^{1}$ Institut de Ciència de Materials de Barcelona-CSIC, Campus UAB, Bellaterra 08193, Spain \\ ${ }^{2}$ Institut Català de Recerca i Estudis Avançats (ICREA), Barcelona, Spain \\ ${ }^{3}$ Departament de Física, Universitat Autònoma de Barcelona, Bellaterra 08193, Spain \\ ${ }^{4}$ Departament de Física Aplicada i Òptica, Universitat de Barcelona, Diagonal 647, Barcelona 08028, Spain \\ ${ }^{5}$ LNMH ONERA-CNRS, BP 4025, 31055 Toulouse, Cedex 4, France
}

(Received 14 July 2006; published 28 November 2006)

\begin{abstract}
The magnetic exchange between epitaxial thin films of the multiferroic (antiferromagnetic and ferroelectric) hexagonal $\mathrm{YMnO}_{3}$ oxide and a soft ferromagnetic (FM) layer is used to couple the magnetic response of the FM layer to the magnetic state of the antiferromagnetic one. We will show that biasing the ferroelectric $\mathrm{YMnO}_{3}$ layer by an electric field allows control of the magnetic exchange bias and subsequently the magnetotransport properties of the FM layer. This finding may contribute to paving the way towards a new generation of electric-field controlled spintronic devices.
\end{abstract}

DOI: 10.1103/PhysRevLett.97.227201

PACS numbers: 75.70.Cn, 85.80.Jm

Multiferroic materials have been proposed for building a new generation of devices in spintronics, eventually allowing us to overcoming critical limitations in technology [1]. Much effort has been directed to searching for materials displaying the elusive coexistence of ferroelectricity (FE) and ferromagnetism (FM) [2,3], which is thought to be essential for progress in this direction. In contrast, materials displaying coupled $\mathrm{FE}$ and antiferromagnetic (AF) behavior have received much less attention. To exploit the multiferroic character of a material, it is essential that the ferroic properties (magnetic and electric, in the present context) are coupled. Hexagonal $\mathrm{YMnO}_{3}$ (YMO), in bulk form, is ferroelectric up to $900 \mathrm{~K}$ and exhibits an antiferromagnetic character at low temperature $\left(T_{N} \sim 90 \mathrm{~K}\right)$. It has been shown that in YMO single crystals, both order parameters are coupled [4], and this observation has triggered a renewed attention to this oxide [5,6]. The electric polarization axis of YMO is along the $c$ axis; the $\mathrm{Mn}$ atomic spins lie in a perpendicular plane, forming a two dimensional, frustrated antiferromagnetic, triangular network $[7,8]$. Hence, in principle, one could use AF YMO to pin the magnetic state of a FM material and subsequently exploit its ferroelectric character and the coupling between $\mathrm{FE}$ and $\mathrm{AF}$ order parameters to tailor the properties of the FM layer. As a first step, it has been recently shown that indeed it is possible to exchange-bias NiFe (Permalloy-Py) with AF epitaxial (0001) YMO films which display a remanent electric polarization [5].

Attempts towards electric-field control of exchange bias have been recently reported by Borisov et al. using magnetoelectric, but not multiferroic (AF) $\mathrm{Cr}_{2} \mathrm{O}_{3}$ single crystals as pinning layers [9]. Here, we will show that it is possible to grow heterostructures that, exploiting the AF and $\mathrm{FE}$ character of $\mathrm{YMO}$, allow us to control the magnetic state of a FM layer by an electric field. For that purpose, an epitaxial layer of YMO has been sandwiched between metallic electrodes ( $\mathrm{Pt}$ and $\mathrm{Py}$ ), and the exchange bias between YMO and Py has been monitored as a function of a biasing electric field applied across the YMO layer [Fig. 1(b)].

When a magnetic field is applied parallel to the interface between FM and AF materials, the magnetization of the FM layer does not follow (neglecting the anisotropy of the FM layer) the external field $\mathbf{H}_{\mathbf{a}}$ but the $\mathbf{H}_{\mathbf{a}}+\mathbf{H}_{\mathbf{e b}}$ vector sum, where $\mathbf{H}_{\mathbf{e b}}$ is the exchange-bias field [10]. The presence of $H_{\mathrm{eb}}$ is commonly evidenced by a shift along the magnetic field axis of the magnetization loop of the FM layer. However, it also dramatically affects the angular dependence of anisotropic magnetoresistance (AMR) of the FM layer when the external magnetic field is rotated [5,11]. If $\theta_{M}$ is the angle between the magnetization (M) and the measuring electric current direction $(\mathbf{J})$, the resistivity is given by $\rho\left(\theta_{M}\right)=\rho_{\perp}+\Delta \rho \cos ^{2} \theta_{M}$, where $\Delta \rho \equiv$ $\rho_{\|}-\rho_{\perp}$ and $\rho_{\perp}$ and $\rho_{\|}$are the resistivity for $\mathbf{M} \perp \mathbf{J}$ and $\mathbf{M} \| \mathbf{J}$, respectively. In the presence of $H_{\mathrm{eb}}$, when rotating the external field $\mathbf{H}_{\mathbf{a}}$ to an angle $\theta_{a}$ with respect to $\mathbf{J}$, the measured $\rho\left(\theta_{a}\right)$, in general, does not follow a simple quadratic $\cos ^{2}\left(\theta_{a}\right)$ dependence. Thus, departure from this behavior allows monitoring the existence of $H_{\mathrm{eb}}$ and its eventual modifications under a biasing electric field.

Hexagonal YMO(0001) films, $90 \mathrm{~nm}$ thick, were grown by pulsed laser deposition on $\mathrm{SrTiO}_{3}$ (111) substrates buffered with a thin epitaxial Pt layer $(8 \mathrm{~nm})$ as bottom metallic electrode. This heterostructure was covered by a Py film $(15 \mathrm{~nm})$. The Pt and YMO films are epitaxial, with (111) and $c$ axis out-of-plane orientation, respectively [5]. Four (inline) electric contacts on Py were used for transport measurements. Additional contacts on $\mathrm{Py}$ and Pt were made for electric biasing of the Py/YMO/Pt sandwich. The resistivity of the YMO layer at $300 \mathrm{~K}$ is of about $10^{6} \Omega \mathrm{cm}$. AMR was measured by rotating an external field $\mathbf{H}_{\mathbf{a}}$ in the plane of the sample by using a physical 

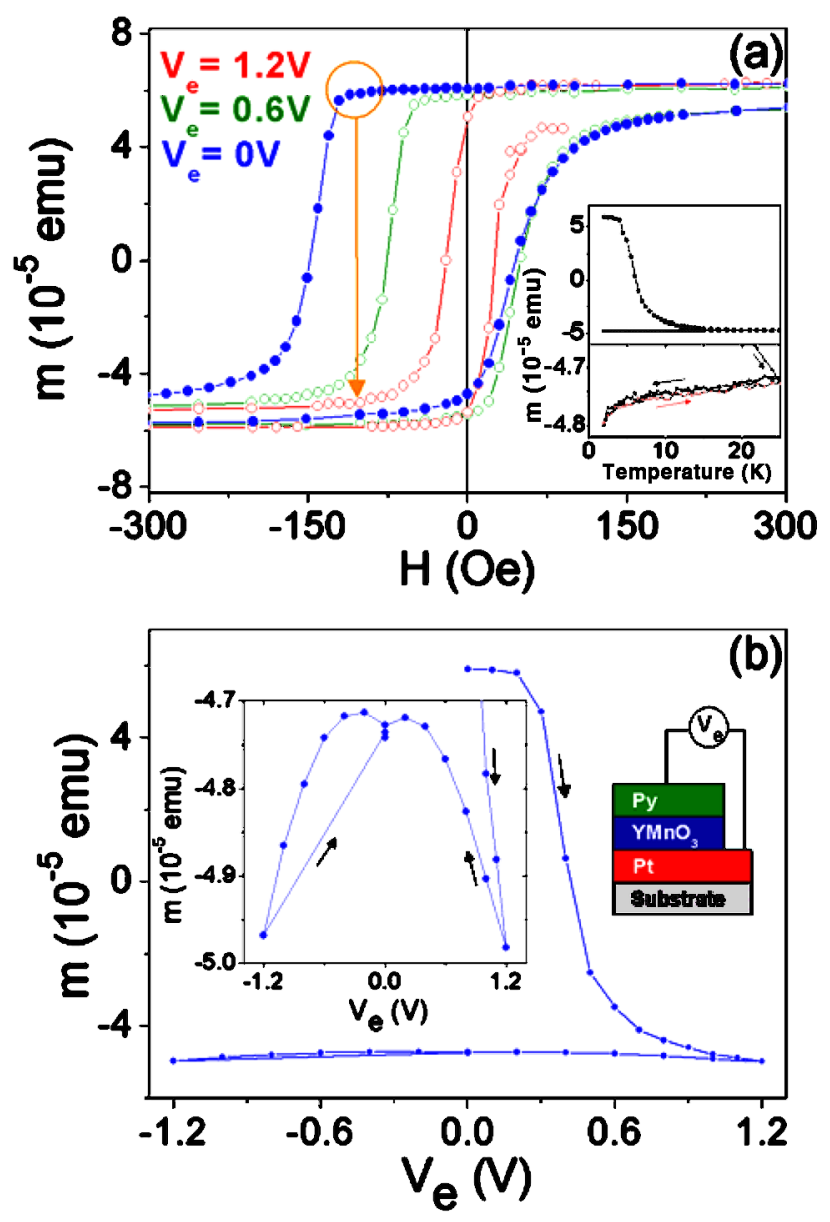

FIG. 1 (color online). (a) Magnetization loops of Py/YMO/Pt, measured at $2 \mathrm{~K}$, after cooling the sample from $300 \mathrm{~K}$ in $3 \mathrm{kOe}$ field, under various biasing-voltage $\left(V_{e}\right)$ values. The circle and arrow illustrate schematically the expected change of magnetization when biasing the sample by an electric field. Inset: temperature dependence of the magnetization at $H=$ $-100 \mathrm{Oe}$ and $V_{e}=0$, when heating the sample from $2 \mathrm{~K}$ to $25 \mathrm{~K}$ (top panel) and subsequent cooling-heating-cooling cycles between $25 \mathrm{~K}$ to $2 \mathrm{~K}$ (bottom panel). (b) Dependence of the magnetization on $V_{e}$ measured at $2 \mathrm{~K}$ in -100 Oe field after cooling the sample from $300 \mathrm{~K}$ in $3 \mathrm{kOe}$ field. Insets: leftzoom of the $+1.2 \mathrm{~V}$ to $-1.2 \mathrm{~V}$ to $0 \mathrm{~V}$ portions of the bias excursion; right - sketch of the sample structure and electric biasing.

properties measurement system from Quantum Design. A SQUID magnetometer was adapted to measure the sample magnetization while biasing it by an electric field.

Figure 1(a) shows the magnetization loops, at $2 \mathrm{~K}$, under various biasing-voltages $\left(V_{e}\right)$, after field cooling ( $\left.3 \mathrm{kOe}\right)$ the sample from $300 \mathrm{~K}$. In the unbiased sample $\left(V_{e}=0\right)$, there is a clear shift of the loop which reflects the existence of an exchange-bias field $H_{\mathrm{eb}}(\approx 60 \mathrm{Oe})$. This observation is compatible with the AF nature of the YMO film below $T_{N}(\sim 90 \mathrm{~K})$. In order to monitor the temperature dependence of $H_{\mathrm{eb}}$, we have measured the temperature depen- dence of the magnetization at $H_{a}=-100 \mathrm{Oe}$, after $3 \mathrm{kOe}$ field cooling. The sample has been heated from $2 \mathrm{~K}$ up to $25 \mathrm{~K}$. The result shown in Fig. 1(a) (inset, top panel) reflects a rapid fall of the magnetization, and thus of the $H_{\mathrm{eb}}$ field, upon heating. The fast reduction of $H_{\mathrm{eb}}$ with increasing temperature results from the low in-plane magnetic anisotropy of the hexagonal YMO [5,12]. Notice in Fig. 1(a) (inset, bottom panel) that, as expected, upon subsequent cooling from $25 \mathrm{~K}$ to $2 \mathrm{~K}$, the magnetization magnitude $(|M|)$ increases.

Data in Fig. 1(a) clearly show that when applying a biasing voltage across the YMO layer, the shift of the $M(H)$ loop gradually disappears, and for $V_{e}=1.2 \mathrm{~V}$, the loop is symmetric and narrower, thus indicating the suppression of $H_{\mathrm{eb}}$ and the coercivity. Inspection of the $M(H)$ loops in Fig. 1(a) suggests that it should be possible to reverse the magnetization of the Py upon suitable electricfield biasing. A dramatic modification of the magnetic response has been indeed measured [Fig. 1(b)] at $2 \mathrm{~K}$, after cooling the sample under $3 \mathrm{kOe}$ field and fixing the measuring field $H_{a}=-100 \mathrm{Oe}$. The arrow in Fig. 1(a) illustrates schematically the process. When increasing $V_{e}$, the magnetization decreases, switches its sign at $V_{e} \approx 0.4 \mathrm{~V}$, and saturates at $V_{e} \approx 1.2 \mathrm{~V}$. Reduction and change of polarity of the electric field does not allow to switch back to magnetization initial $M>0$ value, but it remains $M<0$. This feature can be well appreciated in the zoom shown in Fig. 1(b) (left inset); it is remarkable that upon subsequent reduction of $V_{e}$, the magnitude of $M$ decreases (about $-5 \%$ ), to a minimum value at $V_{e} \approx 0$ and increases again when changing the polarity of the electric field. We notice that this behavior is just opposite to what should be expected if thermal effects associated to Joule heating, virtually unavoidable, were the unique reason for the observed magnetization variation; as illustrated by data in Fig. 1(a) (inset, bottom panel), the magnetization magnitude should keep growing (about $+0.5 \%$ ) when reducing Joule power $\left(V_{e}\right)$. These results indicate that the magnetic exchange bias (and thus the magnetization) can be strongly modified by an electric field.

Further evidence will be gained from magnetotransport data. AMR measurements of Py were performed after field cooling $(3 \mathrm{kOe})$ the sample from $300 \mathrm{~K}$, with the magnetic field applied at $45^{\circ}$ of the measuring current direction. Once at the targeted temperature, the magnetic field is reduced down to the measuring field $H_{a}$ value, and it is afterwards rotated clockwise from $-5^{\circ}$ to $365^{\circ}$ and anticlockwise to the original position $\left(\theta_{a}=0^{\circ}\right.$ corresponds to $\left.\mathbf{J} \| \mathbf{H}_{\mathbf{a}}\right)$ while monitoring the resistance $R\left(\theta_{a}\right)$ as a function of $\theta_{a}$.

Figure 2 collects the $R\left(\theta_{a}\right)$ curves, obtained using $H_{a}=$ $40 \mathrm{Oe}$, at some selected temperatures. Arrows indicate the sense of rotation. We first notice that at $100 \mathrm{~K}, R\left(\theta_{a}\right)$ is well described by a $\cos ^{2} \theta_{a}$ dependence which implies that at this temperature $\theta_{a}=\theta_{M}$ and thus $\mathbf{M}$ is parallel to $\mathbf{H}_{\mathbf{a}}$. 


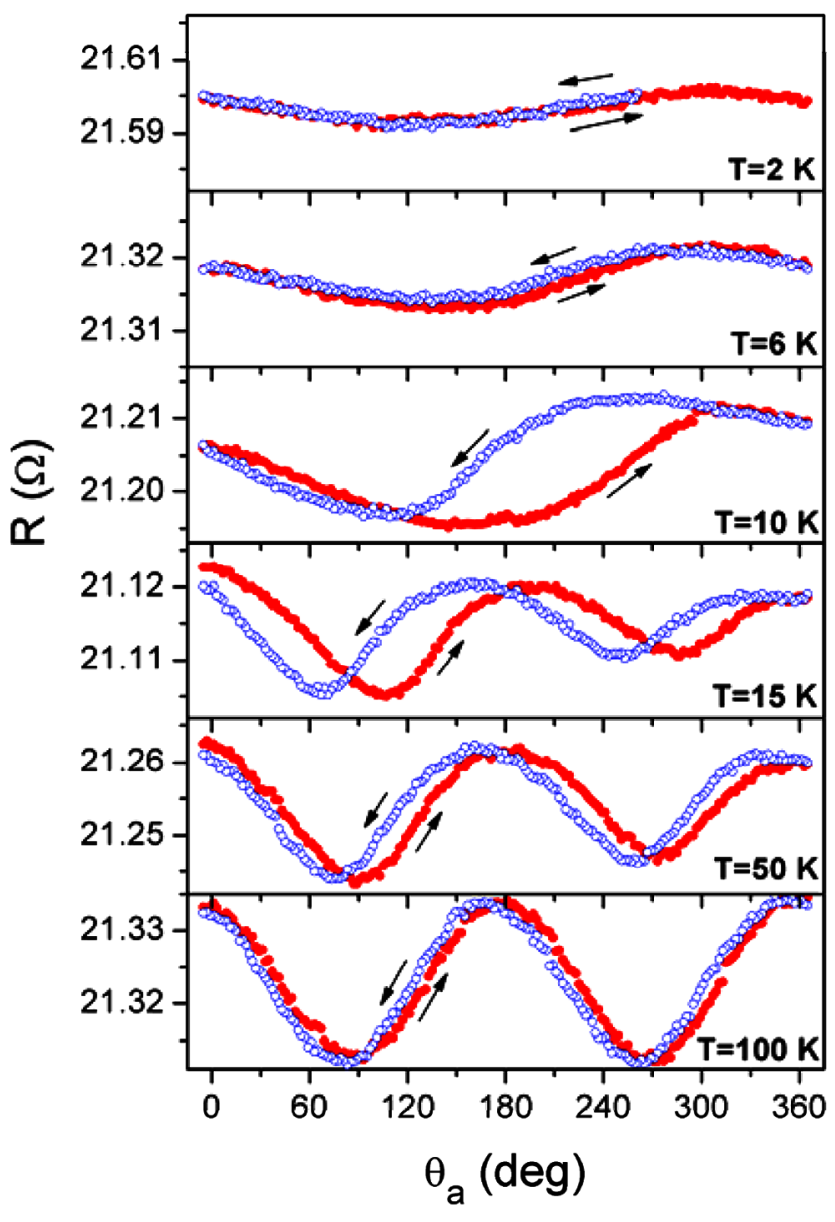

FIG. 2 (color online). AMR of the Py film at different temperatures. Measurements were done in magnetic field of $40 \mathrm{Oe}$, after field cooling ( $3 \mathrm{kOe})$. Similar behavior is observed either just after field cooling from $T>100 \mathrm{~K}$ down to the cited temperatures or at successive heating (after field cooling down to $2 \mathrm{~K}$ ) to selected temperatures in the measuring magnetic field.

Indeed, this is what should be expected as $H_{\mathrm{eb}}$ is zero in the paramagnetic phase of YMO. When reducing the temperature, the $\cos ^{2} \theta_{a}$ dependence disappears, illustrating the presence of $H_{\mathrm{eb}}$. Only one minimum in $R\left(\theta_{a}\right)$, occurring at about $\theta_{a} \sim 120^{\circ}$, remains visible at the lowest temperature. This indicates that at the lowest temperature, under a rotating $H_{a}=40 \mathrm{Oe}$ field, the magnetization remains pinned along the $\mathbf{H}_{\mathbf{e b}}$ direction [5].

We notice that a departure from the $\cos ^{2} \theta_{a}$ dependence still exists at $50 \mathrm{~K}$, indicating a finite $H_{\mathrm{eb}}$ with a strength comparable to the measuring field $\left(H_{a}=40 \mathrm{Oe}\right)$. This observation contrasts with that extracted from magnetization measurements [Fig. 1(a) (inset, top panel)] where the exchange bias is no longer visible in the magnetization loops above $\geq 15 \mathrm{~K}$. The difference is due to the fact that in magnetization measurements, exchange-bias monitoring requires magnetization switching whereas in AMR measurements, this is not required: only small reversible movements of the magnetization around $\mathbf{H}_{\mathbf{e b}}$ can be used to probe the existence of a finite $H_{\mathrm{eb}}$ [11]. This reason also accounts for the absence of hysteresis in measurements performed at the lowest temperature as the magnetic anisotropy is large and $\mathbf{M}$ remains confined within an energy minimum while $\mathbf{H}_{\mathbf{a}}$ rotates; at intermediate temperatures, hysteresis is evident as $\mathbf{M}$ is dephased with respect to $\mathbf{H}_{\mathbf{a}}$. When approaching the Néel temperature, the anisotropy and thus the hysteresis disappear again. In summary, data of Fig. 2 clearly indicate the existence of a finite $H_{\mathrm{eb}}$ field, at least up to $50 \mathrm{~K}$.

In order to explore electric-field effects on $H_{\mathrm{eb}}$ we have measured the AMR response when biasing the $\mathrm{Py} / \mathrm{YMO} / \mathrm{Pt}$ sandwich by an electric field. Figure 3 (top panel) shows the data recorded at $5 \mathrm{~K}$ (after $3 \mathrm{kOe}$ fieldcooling from $300 \mathrm{~K}$ ) at some selected biasing-voltages. The data reveal a strikingly important modification of $R\left(\theta_{a}\right)$. We note for instance, that when increasing $V_{e}$, a second $R\left(\theta_{a}\right)$ minimum at about $\sim 270^{\circ}$ emerges. Comparison of the $R\left(\theta_{a}\right)$ curves in Fig. 2 and 3 (top panel), indicates that upon electric-field biasing the $H_{\mathrm{eb}}$ becomes relatively less relevant on the $R\left(\theta_{a}\right)$ response. That is, the electric field mimics the effect of increasing the temperature (or alternatively, the applied magnetic field $\left.H_{a}\right) . R\left(\theta_{a}\right)$ depends weakly on the $V_{e}$ polarity.

The physical origin of emergence of a minimum of $R\left(\theta_{a}\right)$ at about $\theta_{a} \approx 270^{\circ}$ is illustrated by the diagrams in Fig. 3. Field cooling with field at $\theta_{a}=45^{\circ}$ determines the uniaxial anisotropy axis and defines the exchange-bias field direction. Thus, for small enough $\mathbf{H}_{\mathbf{a}}$ values and for $V_{e}=0$, upon $\mathbf{H}_{\mathbf{a}}$ rotation, $\mathbf{M}$ remains pinned around the anisotropy axis, $\mathbf{M}$ should be at about $\theta_{M} \approx 45^{\circ}$ from $\mathbf{J}$, and $R\left(\theta_{a}\right)$ shows one minimum [Fig. 3(a) and 3(c)]. The appearance of two minima in $R\left(\theta_{a}\right)$, around $\theta_{M} \approx 90^{\circ}$ and $270^{\circ}$ when $V_{e}$ increases, implies that the $\mathbf{M}$ gets closer to $\mathbf{H}_{\mathbf{a}}$, thus recovering the $\cos ^{2} \theta_{a}$ dependence [Fig. 3(b) and 3(d)]. To illustrate the expected modification of $R\left(\theta_{a}\right)$ when reducing the exchange bias, the $R\left(\theta_{a}\right)$ curves in Fig. 3(c) and 3(d) were calculated by using a simple geometrical model to evaluate the angle between $\mathbf{M}$ and $\mathbf{J}$ when rotating $\mathbf{H}_{\mathbf{a}}$ for a fixed $\mathbf{H}_{\mathbf{e b}}$, and subsequently $R\left(\theta_{a}\right)$ [11], for $H_{a} / H_{\mathrm{eb}}=0.1$ and 2 , respectively. The emergence of two minima in $R\left(\theta_{a}\right)$ upon reduction of $H_{\mathrm{eb}}$ is well evident. Therefore, the applied bias voltage reduces the uniaxial exchange-bias based energy barrier.

These results reveal a genuine electric-field effect on the exchange bias in YMO/Py heterostructures. The suppression of magnetic exchange bias by electric poling of the underlying YMO ferroelectric layer indicates a substantial modification of the AF domain structure which is driven by the electric field. The microscopic origin of this effect can not be conclusively inferred from the present experiments. We notice that the (0001) plane of the YMO is a compensated spin-lattice. Although exchange bias in compensated surfaces is well documented, [10] its origin is still under debate. It has been proposed that exchange-bias in com- 

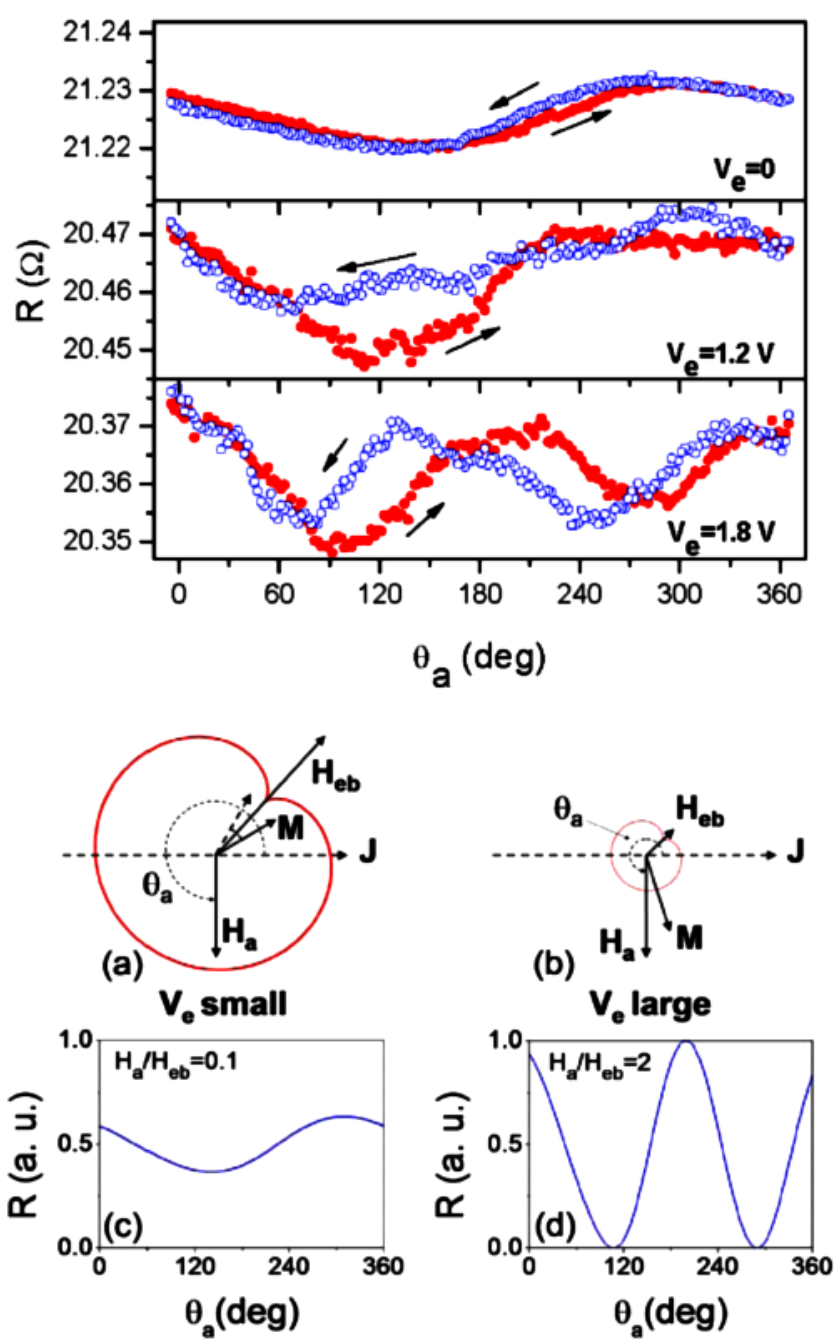

FIG. 3 (color online). Top panel: AMR of the Py film when biasing the $\mathrm{Py} / \mathrm{YMO} / \mathrm{Pt}$ sandwich by an electric field. Measurements were done at $5 \mathrm{~K}$ in magnetic field of $50 \mathrm{Oe}$, after field cooling $(3 \mathrm{kOe})$ and applying $V_{e}=0,1.2$, and $1.8 \mathrm{~V}$ bias voltages. Bottom panels: Scheme of the uniaxial in-plane magnetic anisotropy for small (a) and large (b) electric bias field. Pinning of $\mathbf{M}$ around $\mathbf{H}_{\mathbf{e b}}$ is illustrated by the small dashed arrow in (a). Solid lines qualitatively indicate the in-plane anisotropy energy due to $\mathbf{H}_{\mathbf{e b}}$ field, more relevant in (a) than in (b). The orientation of the $\mathbf{M}$ with respect to the measuring field $\left(\mathbf{H}_{\mathbf{a}}\right)$, the field-cooling direction $\left(\mathbf{H}_{\mathbf{e b}}\right)$, and the current $(\mathbf{J})$ are displayed. Panels (c) and (d) show the AMR response calculated as described in the text.

pensated interfaces could be controlled by domain-wall (DW) formation and pinning [13]. This suggestion combined with the observation that $\mathrm{FE}$ and $\mathrm{AF}$ domains are clamped [4] provides a natural framework to understand the observed effects: pinning and/or creation of AF DW at the position of the FE DW, owing to the reduction of the total energy of the crystal, could contribute also to the exchange bias. Washing out the FE domains by applying sufficiently high electric field would result in reducing or vanishing of the $H_{\mathbf{e b}}$ and a weak dependence on polarity. Both features are observed in our experimental data. We further suggest that similar experiments to those reported here, but performed using biferroic ( $\mathrm{AF}$ and $\mathrm{FE}$ ) oxides having a larger magnetic anisotropy, would allow further improvement of the response and higher operation temperature.

In summary, we have shown that an electric field can be used to tune the exchange-bias coupling in AF/FM heterostructures and eventually the magnetic switching of the FM layer. Here, we have used the biferroic antiferromagnetic and ferroelectric YMO layer to bias the FM layer, with the ultimate goal of fully exploiting its ferroelectric character and subsequent hysteretic behavior. The present results illustrate the promising perspectives of $\mathrm{AF} / \mathrm{FE}$ oxides in spintronics and may have impact on a new generation of electric-field controlled magnetic devices.

Financial support by the MEC of the Spanish Government (Projects Nos. NAN2004-9094-C03 and MAT2005-5656-C04) and by the EU [Project MaCoMuFi (No. FP6-03321) and FEDER] and by the Generalitat de Catalunya (Project 2005SGR00509) are acknowledged. The authors are thankful to J. M. Pérez for technical support.

[1] Ch. Binek and B. Doudin, J. Phys. Condens. Matter 17, L39 (2005); M. Fiebig, J. Phys. D: Appl. Phys. 38, R123 (2005).

[2] N. A. Hill, J. Phys. Chem. B 104, 6694 (2000).

[3] M. Gajek et al., Phys. Rev. B 72, 020406 (2005).

[4] M. Fiebig et al., Nature (London) 419, 818 (2002).

[5] X. Martí et al., Appl. Phys. Lett. 89, 032510 (2006).

[6] J. Dho and M. G. Blamire, Appl. Phys. Lett. 87, 252504 (2005).

[7] See for instance B. B. van Aken et al., Nat. Mater. 3, 164 (2004).

[8] A. Muñoz et al., Phys. Rev. B 62, 9498 (2000).

[9] P. Borisov et al., Phys. Rev. Lett. 94, 117203 (2005).

[10] J. Nogués and I. K. Schuller, J. Magn. Magn. Mater. 192, 203 (1999).

[11] B. H. Miller and E. Dan Dahlberg, Appl. Phys. Lett. 69, 3932 (1996); H. L. Brown et al., J. Appl. Phys. 91, 7415 (2002).

[12] M. Fiebig et al., Phys. Rev. B 65, 224421 (2002).

[13] A.P. Malozemoff, J. Appl. Phys. 63, 3874 (1988); U. Nowak, A. Misra, and K. D. Usadel, J. Appl. Phys. 89, 7269 (2001); D. Suess et al., Phys. Rev. B 67, 054419 (2003). 